\title{
Comparison of prescribing and dispensing processes between veterinarians and pharmacists in New Zealand: Are there opportunities for cooperation?
}

\author{
Arlene McDOWELL, Lauren ASSINK, Rebecca MUSGRAVE, Hannah SOPER,
} Chantal WILLIAMS, Pauline NORRIS.

Received (first version): 24-Aug-2010 Accepted: 29-Dec-2010

\begin{abstract}
${ }^{*}$
Background: Prescribing and dispensing of medicines are fundamental processes in providing healthcare for both human and animal patients. There has been recent discussion in the literature to advocate for increased co-operation between pharmacists and veterinarians, however there is little data available about veterinary prescribing and dispensing processes.

Objective: The aims of this study were to gain information on veterinary prescribing and dispensing processes for companion animals in the Dunedin region of New Zealand.

Methods: Open interviews were conducted with a selection of five veterinarians at practices in Dunedin. All interviews were transcribed verbatim. Results: In New Zealand almost all dispensing of medicines for animals is carried out by veterinarians or their staff. There is a lack of standard treatment guidelines and a lack of regulation around the treatment period for which medicines can be dispensed at one time (i.e. period of supply). Medicines for animals are sometimes dispensed by community pharmacies, where clients are experiencing financial difficulties or when particular medicines are not held by veterinary practices. Record keeping requirements and practices for veterinarians are similar to those for community pharmacies.

Conclusions: Processes undertaken by veterinarians in terms of prescribing and dispensing were similar to pharmacists' practices for human patients and so there is opportunity for collaboration between the two professions. Pharmacists also have complementary knowledge about dosing and formulating medicines that can assist in delivering optimal healthcare to animal patients.
\end{abstract}

\footnotetext{
Arlene McDOWELL. PhD. Senior Lecturer in Pharmaceutical Science. University of Otago. Dunedin (New Zealand).

Lauren ASSINK. BPharm. School of Pharmacy, University of Otago. Dunedin (New Zealand).

Rebecca MUSGRAVE. BPharm. School of Pharmacy, University of Otago. Dunedin (New Zealand).

Hannah SOPER. BPharm. School of Pharmacy,

University of Otago. Dunedin (New Zealand).

Chantal WILLIAMS. BPharm. School of Pharmacy,

University of Otago. Dunedin (New Zealand).

Pauline NORRIS. PhD. Professor of Social Pharmacy.

University of Otago. Dunedin (New Zealand).
}

Keywords: Pharmacists. Veterinarians. Interprofessional Relations. New Zealand.

\section{COMPARACIÓN DE LOS PROCESOS DE PRESCRIPCIÓN Y DISPENSACIÓN ENTRE VETERINARIOS Y FARMACÉUTICOS EN NUEVA ZELANDA: HAY OPORTUNIDADES DE COOPERACIÓN?}

\section{RESUMEN}

Antecedentes: La prescripción y la dispensación de medicamentos son procesos fundamentales en la provisión de cuidados de salud tanto para humanos como para animales. Recientemente se ha discutido en la literatura para abogar por el aumento de cooperación entre farmacéuticos y veterinarios, sin embargo hay pocos datos disponibles sobre los procesos de la prescripción y la dispensación veterinaria.

Objetivo: Los objetivos de este estudio fueron obtener información sobre los procesos de prescripción y dispensación veterinaria en animales de compañía en la región de Dunedin en Nueva Zelanda.

Métodos: Se realizaron entrevistas abiertas a una selección de cinco veterinarios en clínicas de Dunedin. Todas las entrevistas se transcribieron literalmente.

Resultados: En Nueva Zelanda, casi todas las dispensaciones de medicamentos para animales son realizadas por veterinarios o su personal. Hay una falta de guías de tratamientos estandarizados y de legislación sobre el periodo de tratamiento que puede ser dispensado cada vez (i.e. periodo de provisión). Los medicamentos para animales a veces se dispensan en farmacias comunitarias, cuando los clientes sufren dificultades financieras o cuando las clínicas veterinarias carecen de ellos. Los requisitos de registro y las prácticas de los veterinarios son similares a los de las farmacias comunitarias.

Conclusiones: Los procesos realizados por veterinarios para la prescripción y dispensación fueron similares a las prácticas para pacientes humanos, así que hay una oportunidad de colaboración entre las dos profesiones. Los farmacéuticos pueden tener conocimientos complementarios sobre dosificación y formulación de los medicamentos que pueden ser de ayuda para proporcionar los cuidados de salud óptimos para los pacientes animales. 
Palabras clave: Farmacéuticos. Veterinarios.

Relaciones interprofesionales. Nueva Zelanda.

\section{INTRODUCTION}

Veterinarians are specialists in the health and well being of animals. The role of the veterinarian is both broad and diverse, encompassing the treatment of sick and injured animals and surgical services. In addition to this, veterinarians are also involved in numerous other aspects of animal care such as the prevention of animal ailments by administering immunizations, de-sexing of animals and animal lifestyle advice. Veterinary practices can also impact the wider community through biosecurity and in the area of public health through involvement in zoonotic disease control. In New Zealand, by law (Veterinarians Act 2005) a veterinarian is a person who is registered with the Veterinarian Council of New Zealand and holds a current practicing certificate. Eligibility for registration requires the individual to have a recognized veterinary science degree or diploma from a recognized institution or pass a prescribed examination or assessment; meet minimum fitness practice requirements and ability to communicate in English. Within the veterinary profession, there is a division between the treatment of food production animals and companion animals. ${ }^{1}$ The two areas of veterinary practice overlap to some degree, yet also differ in the number and type of animals being treated, and the involvement within society, particularly public health. The present study will focus on practices applicable to companion animals.

Pharmacists are health professionals that are highly regarded as the custodians of medicines within both a community and clinical setting. Traditionally pharmacists primarily distributed drugs that were prescribed by a medical practitioner for human patients. Now the role of the pharmacist has become much more diverse. This diversification now sees pharmacists involved in a more expansive range of responsibilities with respect to patient care; from providing information on the selection, dosage, interactions and side effects of medications through to provision of on-going monitoring of patients and the suitability of their medication regime.

Veterinarians and pharmacists share a desire to achieve the maximal health outcome for their respective patients. The power to treat an injured or unwell animal rests in the hands of the veterinarian in terms of issuing a prescription ${ }^{2}$ or with the owner in terms of deciding on the most appropriate treatment option for the animal under their care. Whilst the pharmacist is legally unable to participate in the process of issuing a prescription to an animal, they are able to be involved in formulating, compounding and dispensing of veterinary prescriptions. Pharmacists are also able to provide information on prophylaxis for a number of diseases and advice on the best possible treatment options available. In recent years there has been a resurgence of interest in the discipline of veterinary pharmacy ${ }^{3}$ and the potential role that pharmacists can play in the provision of drug therapies for animal patients. ${ }^{4}$ The delivery of more cost-effective medicines may also be possible if animal products are dispensed through a pharmacy. On the basis of reducing costs, a government report compiled for the Minister of Agriculture, Fisheries and Food in the United Kingdom recommended that veterinarians should use pharmacies to dispense prescriptions. ${ }^{5}$ The present study aimed to explore the veterinarians' practices with regard to the prescribing and dispensing of veterinary medicines and investigate whether pharmacists could become involved in these processes. Previous studies have looked at prescribing patterns of different drug classes, in particular antimicrobials for production animals ${ }^{6}$, however no published studies have explored the potential link between veterinary prescribing and dispensing practices and the involvement of pharmacists.

\section{METHODS}

Data was collected through focused discussions with either the veterinarian or veterinary nurse from the Dunedin region of New Zealand. Veterinarians were approached from seven urban practices and invited to participate in a semi-structured interview. Of the practices contacted, five practices indicated willingness to participate in this study. The study focused on veterinary requirements for domestic animals and all herd animal data was excluded from this study.

Interviews took around 15-20 minutes and focused on five main topics and all participants were asked the same questions by one of two pairs of interviewers. The questions were around the themes of veterinary records, general treatment, prescriptions, antibiotics and drug supply, with specific questions directed to allow comparisons to be made with the pharmacy profession (Table 1). The discussion utilized an open questioning technique. Participants were encouraged to participate actively in open discussion but could decline to answer any questions. Extensive notes were taken during all interviews and for each interview both a moderator and note-taker were present.

\section{Data Analysis}

Analysis began with all notes being read to get a general impression of all the collected material. The data from the individual veterinary practices was then compared and contrasted in order to collate the common ideas and themes resulting from the discussion.

\section{Ethical Consideration}

Ethical approval was obtained from the University of Otago prior to the commencement of the study. Each participant was given an information sheet outlining the aim of the project, participants and their role in the study. They were also asked to sign a consent form stating their consent was voluntary and participants were free to withdraw from the project at any time. 


\begin{tabular}{|l|}
\hline Table 1. Questions asked during the interview with staff from the veterinary practices. \\
\hline Records \\
\hline How long are records kept? \\
\hline Where are the records kept? \\
\hline How are the records categorised? \\
\hline Do you have a consultation recording guideline? \\
\hline Can an animal's previous consult be referred to? \\
\hline Is there standard veterinary record dispensing software? \\
\hline General animal/treatment \\
\hline What are the majority of animals that come in? E.g dog, cat? \\
\hline Is there a procedure / guideline that you follow for each consultation? \\
\hline Is there a standard treatment guideline for some conditions? \\
\hline Prescriptions \\
\hline What is the period of supply of a veterinary prescription? \\
\hline Is there a dosing nomogram for animals (mg/kg) and where does one look up that information? \\
\hline Where do vets compound and dispense veterinary medicines? \\
\hline Where would you find additional information about drugs, and also about specific conditions? \\
\hline Do you have involvement with Pharmacists? If yes, in regards to what? \\
\hline What are the most common problems with animals coming in, and seasonal variations? \\
\hline What is the role of the vet nurse with regard to the animal care, including prescribing and dispensing? \\
\hline Antibiotics \\
\hline What proportion of consultations results in an antibiotic prescription? \\
\hline What is the most common antibiotic given and what is its dose and formulation? \\
\hline Drug supply \\
\hline Where do you buy medicines? \\
\hline Do drug sales representatives visit you? \\
\hline How do you decide what to buy and how much to buy? \\
\hline Who decides what is purchased? \\
\hline
\end{tabular}

\section{RESULTS}

Five veterinary practices approached agreed to participate in this study. Participants had different levels of veterinary experience and included both male and female veterinarians. The majority of animals treated in these urban clinics were cats and dogs, with other small animals being treated less frequently (Figure 1). All veterinary practices in the study obtained medicines from veterinary product wholesalers who also stock human medicines.

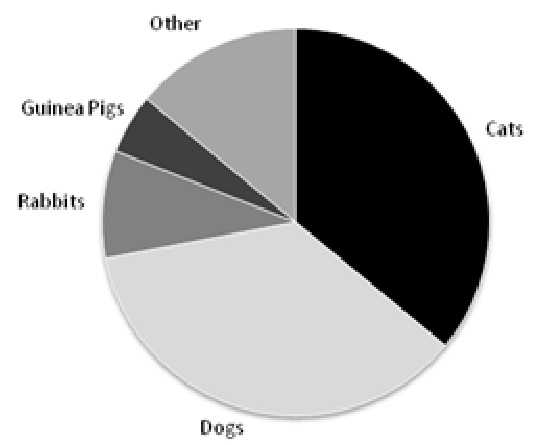

Figure 1. Proportion of domestic animal species most frequently examined by veterinarians from five veterinary practices in the Dunedin region of New Zealand $(n=5)$. Animals included in the category "other" were birds, rats, goats and pigs.

\section{Prescribing}

The most popular literature for information on medications, prescribing and dosing procedures was Plumb's Veterinary Drug Handbook with each practice having access to at least one copy. Other literature referred to was the Index of Veterinary Specialities (IVS) Annual, a comprehensive online database of medicines and products for the New Zealand animal health industry. Another source of information was the charts and packaging data from pharmaceutical companies who often supplied nomograms and formulas for drug dosage calculations.

Veterinarians reported that they took owners' financial situation into account when prescribing and dispensing. Where possible and (or) appropriate veterinarians would dispense the most cost effective option or write a prescription to be filled at a pharmacy.

The veterinarians who participated in the study were not aware of any legislation that restricted the period for which medicine was supplied and acknowledged that they were unaware of guidelines preventing them from doing so. They reported that the amount of medicine they supplied depended on ethical considerations and the financial resources of clients. Despite the lack of regulations, the veterinarians had established their own guidelines. Most generally supplied a quantity sufficient for a period of one month of treatment, or for ongoing conditions, a period of six months of treatment. In chronic cases, such as thyroid problems, a check up was required every six months to assess the animal's progress and condition. For more acute conditions such as an infection, the medication provided was generally for a shorter period of supply such as for one to two weeks. By selfregulating the period of supply, the veterinarians were able to ensure that the animal was receiving the appropriate treatment and care necessary and not encouraging irrational use of medicines. This was especially important for medicines with a high abuse potential such as phenobarbitone. 


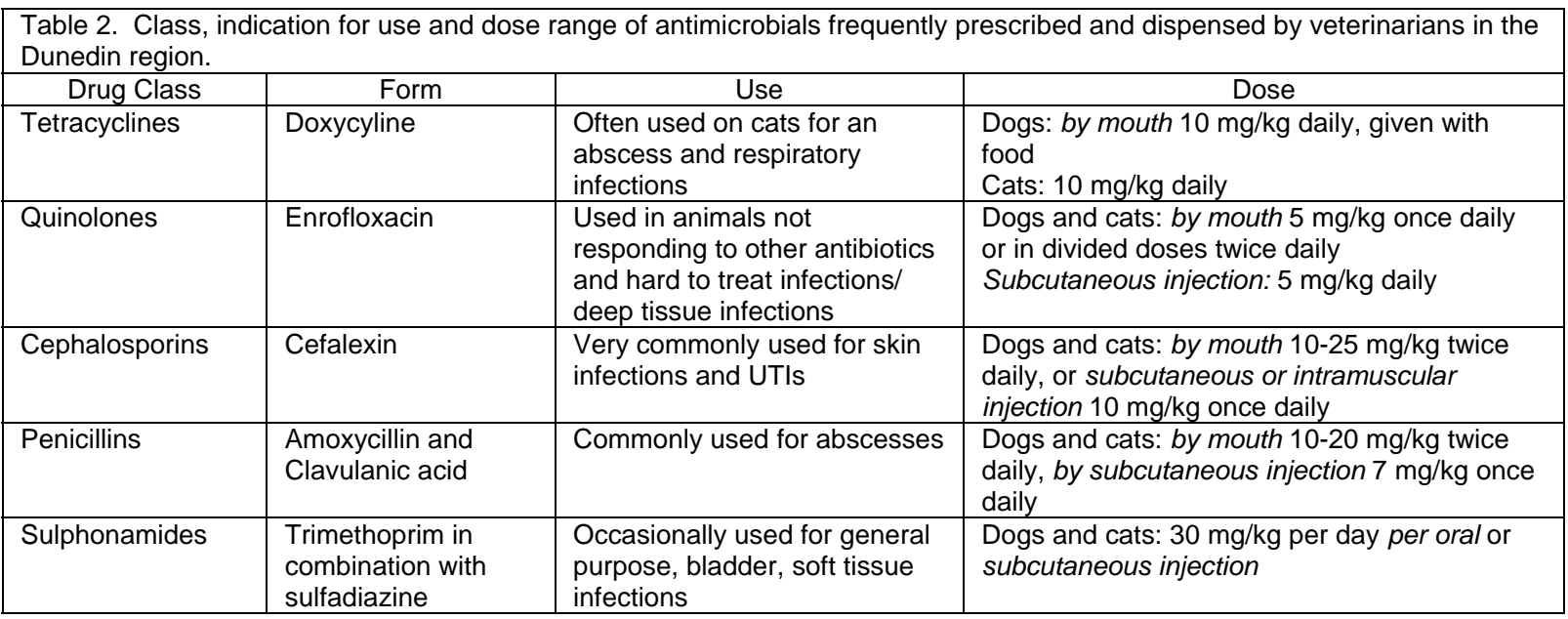

Antibiotic usage in veterinary medicine was varied throughout the different veterinary practices with an estimated $60 \%$ of consultations resulting in an antibiotic (Table 2), however individual practices varied widely, from an estimate of $25 \%$ to $85 \%$. Vaccinations were reported to be the second most commonly administered medication during an animal consultation at the veterinary practices visited.

\section{Dispensing}

Most veterinarians reported that they physically dispensed medications themselves, including selection of the medicine from the supply area, packaging the appropriate quantity and labeling the medicine before giving it to the animal/owner, whilst veterinary nurses usually prepared animals for consultation and procedures. However in some cases it was veterinary nurses often undertook the dispensing process and dispensed routine medications after communication with the veterinarian. Two of the veterinary practices reported compounding medications in their own clinics and others used a compounding pharmacy (Optimus Healthcare Limited in Auckland, New Zealand).

Although it is not common practice for veterinarians to write prescriptions to be filled in community pharmacies, it does occur in specific situations. The cost of medicines dispensed by pharmacies is usually lower than the cost at the veterinary clinic. 5 If the veterinarian is aware of financial hardship with their clients, then they may take this into consideration and opt to write a prescription for the client to take to a community pharmacy. While veterinarians acknowledged that it was not routine practice, they agreed that they would rather the animal have access to the medication. All five veterinary practices interviewed stated that the reason for prescriptions being written by veterinarians was if the patient required a medicine that the veterinary practice did not have in stock, or a medicine that was not commonly used (e.g. a chemotherapy drug).

\section{Relationships with pharmacists}

All five veterinary practices consulted reported involvement with pharmacists as part of their prescribing and dispensing processes, however two practices noted that this contact was on an occasional basis. The reasons reported for the interaction with pharmacists was when a drug was not available as a veterinary formulation, a prescription would be written and taken to a pharmacy for dispensing, pharmacists were consulted to see if there was a cheaper generic product available from the pharmacy.

\section{Record-keeping}

Veterinary records for the animal patients were kept electronically for at least 10 years (VisionVPM Veterinary Practice Management software Records software, Provet IT New Zealand, Tauranga) and in most practices were permanent. In some practices the records were kept for the animal's lifetime and as well as a couple of years after the animal died. The records were all kept electronically and were most commonly categorized under the human owner's name with the animals' name as a subcategory. Other veterinary practices preferred to categorize their records under the animals' name but using the owner's surname, allowing retrieval of records for more than one animal living in the same human family. In one practice, if laboratory tests were authorised, the results were scanned and attached to animals' individual electronic record. All practices reported having access to records from previous consultations.

Records are valuable sources of information as well as legal documents, it is therefore imperative to utilize and maintain easily accessible, organised records. Findings indicated that like pharmacy records, veterinary records were categorized primarily under the clients' name and included a patient history. In both disciplines records are stored electronically with the use of practice-based computer programs designed specifically for use in either pharmacy or veterinary practice. Because of their dual role as prescribers and dispensers for 
animals, veterinary software was often more complex than pharmacy dispensing software, because it includes medical history as well prescribing history. Like pharmacy dispensing software, veterinary programs allow for the construction of labels for medication. Unlike pharmacy dispensing software, veterinary programs included fewer features like interaction prompting and essential medical information. The prescribing veterinarian must also keep records of each prescription issued for five years and these should be cross-referenced to the details of the associated veterinary consultation (ACVM Standard for Prescription Animal Remedy Veterinary Medicines, Section 2.4.3). This is in contrast to a pharmacy where prescriptions must be stored for 10 years, on site for three years in original form and then for a further seven years to comply with legal standards (Health (Retention of Health Information) Regulations SR1996/343). In both veterinary clinics and pharmacies, records were kept a minimum of 10 years and were usually maintained for the life of the patient, this complies with Section 45 of the Medicines Act 1981, which states that every person in a business who supplies medicine must keep records for such period as may be prescribed.

Veterinary prescribing is the process of selecting a suitable medication and treatment regime tailored to the animal's needs. Legally, medicines that are classified as 'prescription only' may only be dispensed by a veterinarian or pharmacist following a clinical assessment by the veterinarian and may only be dispensed or sold in accordance with a prescription. Although it is not common practice for veterinarians to write prescriptions to be filled in community pharmacies, it does occur in specific situations. In many instances it is more economical for the patient if the veterinarian writes a prescription, as the cost of the medicine dispensed by pharmacies is usually lower than the cost at the veterinary clinic. 5 If the veterinarian is aware of financial hardship with their clients, then they may take this into consideration and opt for this course of action. While veterinarians acknowledged that it was not routine practice, they agreed that they would rather the animal have access to the medication. From our observations, the most common reason for prescriptions being written by veterinarians was if the patient required a medicine that the veterinary practice did not have in stock, or a medicine that was not commonly used.

Although veterinarians and their staff performed almost all dispensing, community pharmacies were sometimes used. There were two main reasons for this: lower price and wider range of products available. Pharmacies stock a more extensive range of medicines than veterinary practices, due to the vast array of human medical conditions requiring treatment. The most likely reason for this difference is the government regulatory body (PHARMAC) funds many high-demand human medicines in New Zealand, whereas veterinary medicines are funded directly by the consumer.

There appear to be no regulations or guidelines about the period of supply for medicines dispensed by veterinarians. However, when veterinarians prescribe medicines to be dispensed by pharmacists, there is legislation regarding the period of supply. This is due to a legal difference between an "authorization" and a "prescription" of a veterinary medicine. When veterinarians, or their staff, dispense medicines this is referred to as an authorization (a documented instruction authorizing the sale or use of a medicine by a veterinarian (Agricultural Compounds and Veterinary Medicines (ACVM) Standards for Prescription Animal Remedies, Section 2.4.3). A prescription refers to a documented instruction to dispense a medication and is usually intended for pharmacists or traders. In the latter case, the medicine must be dispensed within 30 days of the date of writing and the prescription must specify a period of treatment, not exceeding six months (ACVM) Standards for Prescription Animal Remedies, Section 2.4.3). Thus there are more strict requirements for medicines dispensed to animals by community pharmacists compared with those dispensed by veterinarians. Medicines dispensed to human patients are further restricted by legislation. Doctors are restricted to prescribing a maximum of three months supply of a medicine at any one time. Depending on the medication prescribed and the funding requirements, a pharmacist may dispense the total three months supply at once, or as a monthly dispensing. Controlled drugs for humans have their own requirements. These can be prescribed for a maximum of one months supply and dispensed in quantities sufficient for either one week or 10 days treatment.

Our study concluded that veterinarians in this region themselves did the majority of the dispensing including selection of the medicine from the supply area, packaging the appropriate quantity and labeling the medicine before giving it to the animal/owner. In some instances, veterinary nurses would aid in the dispensing of routine medication, under the direction of the veterinarian. Legally an agent or employee at a veterinary practice may dispense a prescription medicine for the treatment of an animal under the direction of the veterinary surgeon (Medicines Regulations (SR 1984/143) 7 August 2009; Regulation 42 (2)). This is in contrast to the tighter regulations in place in community pharmacy. Dispensary technicians, pharmacy graduates, pharmacy technicians and students can only dispense prescription medicines under the direct supervision of a pharmacist (Medicines Regulations (SR 1984/143) 7 August 2009; Regulation $42(1 \mathrm{~A})$ ) and all of their actions must be authorized as correct before the medication can leave the dispensary. Pharmacists also have ethical responsibilities to check that the doses are correct and the prescribed medicine is appropriate for the patient, before dispensing the medicine. If there is any uncertainty with these aspects or any component of the prescription, the pharmacist is obliged to contact the prescriber. In veterinary practice, as it is the veterinarian who prescribes the medicine and does the majority of the dispensing, they are responsible for ensuring that the dose and medicine is appropriate for their patients. Thus, for animals, the veterinarian carries out the roles of 
both doctor and pharmacist, which means there is no verification of dosage or suitability by an independent source. Pharmacists are in a suitable environment to provide medicines to the owners of animals, as they have both knowledge of medicines and access to supplies. Although we observed that it was not a common practice, there is definite potential for pharmacists to play a role in the veterinary field, as has been suggested by other authors. $^{4,7}$

In Dunedin, both veterinarian clinics and pharmacies source the majority of their medicines from the same supplier. This wholesaler does not restrict each professions to obtaining only medicines specific to their line of work, as both professions have access to both human and veterinary medicines. Where a product is not readily accessible, the veterinarians and pharmacists must either compound the product, order a suitable formulation or change the medication. The general consensus is that on-site compounding is being phased out in both professions in favor of manufactured formulations that can be ordered through compounding companies. In the case of veterinary medicine, compounding may be ordered specific to the patient through companies such as Optimus, a compounding pharmacy that specializes in extemporaneous compounding of both veterinary and human medicine. Pharmacists on the other hand are frequently required to compound these items for individual patients if the product is not readily available.

The common classes of antimicrobials prescribed in a veterinary setting (Table 2 ) are also routinely prescribed for use in humans and are regularly dispensed in community pharmacies. This highlights the potential for pharmacist involvement in veterinary medicine with the provision of a more affordable and convenient service for animal owners. The emergence of antimicrobial resistance emphasizes the importance of ensuring the correct and appropriate use of antibiotics by veterinarians. ${ }^{8}$

With respect to labeling of medicines, the pharmacist must maintain a thorough, succinct process to produce a label adequate to the patient for both legal and ethical requirements. In veterinary practice, this process is less regulated with the labels being made either by the veterinarian at the time of dispensing or authorization or by verbal sanctioning to veterinary nurses. Labeling of veterinary medicine has additional requirements to prescribed medicines intended for human use. Table 3 shows the common requirements that are requisites for the labeling of medicines in both professions, and additional information that must be included in the veterinary setting. The difference evident with labeling of medicines intended for animals is the inclusion of the terms "For Animal Treatment Only" and "Keep Out of Reach of Children". This could be easily incorporated into the label when dispensed in the pharmacy setting, and is not a significant barrier that would prevent large quantities of animal medicines from being dispensed in a community pharmacy.

\section{DISCUSSION}

Records are valuable sources of information as well as legal documents, it is therefore imperative to utilize and maintain easily accessible organised records. Findings indicated that like pharmacy records, veterinary records were categorized primarily under the clients' name and included a patient history. In both disciplines records are stored electronically with the use of practice-based computer programs designed specifically for use in either pharmacy or veterinary practice. Because of their dual role as prescribers and dispensers for animals, veterinary software was often more complex than pharmacy dispensing software, because it includes medical history as well prescribing history. Like pharmacy dispensing software, veterinary programs allow for the construction of labels for medication. Unlike pharmacy dispensing software, veterinary programs included fewer features like interaction prompting and essential medical information. The prescribing veterinarian must also keep records of each prescription issued for five years and these should be cross-referenced to the details of the associated veterinary consultation (ACVM Standard for Prescription Animal Remedy Veterinary Medicines, Section 2.43). This is in contrast to a pharmacy where prescriptions must be stored for 10 years, on site for three years in original form and then for a further seven years to comply with legal standards (Health (Retention of Health Information) Regulations SR1996/343). In both veterinary clinics and pharmacies, records were kept a minimum of 10 years and were usually maintained for the life of the patient, this complies with Section 45 of the Medicines Act 1981, which states that every person in a business who supplies medicine must keep records for such period as may be prescribed.

Veterinary prescribing is the process of selecting a suitable medication and treatment regime tailored to the animal's needs. Legally, medicines that are classified as 'prescription only' may only be dispensed by a veterinarian or pharmacist following a clinical assessment by the veterinarian and may only be dispensed or sold in accordance with a

\begin{tabular}{|l|l|}
\hline Table 3. Legal labeling requirements for inclusion on veterinary products. & \\
\hline Essential requirements regardless of profession & $\begin{array}{l}\text { Additional veterinary } \\
\text { labels required }\end{array}$ \\
\hline - Dispensing practice name, address and contact phone number & - "For Animal Use Only" \\
- Name of prescriber & $\begin{array}{c}\text { "Keep out of reach of } \\
\text { Children" }\end{array}$ \\
- Patient name & \\
- Product name and strength & \\
- Quantity & \\
- Date dispensed & \\
- Esirections for use & \\
\hline
\end{tabular}


prescription. Although it is not common practice for veterinarians to write prescriptions to be filled in community pharmacies, it does occur in specific situations. In many instances it is more economical for the patient if the veterinarian writes a prescription, as the cost of the medicine dispensed by pharmacies is usually lower than the cost at the veterinary clinic. ${ }^{5}$ If the veterinarian is aware of financial hardship with their clients, then they may take this into consideration and opt for this course of action. While veterinarians acknowledged that it was not routine practice, they agreed that they would rather the animal have access to the medication. From our observations, the most common reason for prescriptions being written by veterinarians was if the patient required a medicine that the veterinary practice did not have in stock, or a medicine that was not commonly used.

Although veterinarians and their staff performed almost all dispensing, community pharmacies were sometimes used. There were two main reasons for this: lower price and wider range of products available. Pharmacies stock a more extensive range of medicines than veterinary practices, due to the vast array of human medical conditions requiring treatment. The most likely reason for this difference is the government regulatory body (PHARMAC) funds many high-demand human medicines in New Zealand, whereas veterinary medicines are funded directly by the consumer.

There appear to be no regulations or guidelines about the period of supply for medicines dispensed by veterinarians. However, when veterinarians prescribe medicines to be dispensed by pharmacists, there is legislation regarding the period of supply. This is due to a legal difference between an "authorization" and a "prescription" of a veterinary medicine. When veterinarians, or their staff, dispense medicines this is referred to as an authorization (a documented instruction authorizing the sale or use of a medicine by a veterinarian (Agricultural Compounds and Veterinary Medicines (ACVM) Standards for Prescription Animal Remedies, Section 2.4.3). A prescription refers to a documented instruction to dispense a medication and is usually intended for pharmacists or traders. In the latter case, the medicine must be dispensed within 30 days of the date of writing and the prescription must specify a period of treatment, not exceeding six months (ACVM) Standards for Prescription Animal Remedies, Section 2.4.3). Thus there are more strict requirements for medicines dispensed to animals by community pharmacists compared with those dispensed by veterinarians. Medicines dispensed to human patients are further restricted by legislation. Doctors are restricted to prescribing a maximum of three months supply of a medicine at any one time. Depending on the medication prescribed and the funding requirements, a pharmacist may dispense the total three months supply at once, or as a monthly dispensing. Controlled drugs for humans have their own requirements. These can be prescribed for a maximum of one months supply and dispensed in quantities sufficient for either one week or 10 days treatment.
Our study concluded that veterinarians in this region themselves did the majority of the dispensing including selection of the medicine from the supply area, packaging the appropriate quantity and labeling the medicine before giving it to the animal/owner. In some instances, veterinary nurses would aid in the dispensing of routine medication, under the direction of the veterinarian. Legally an agent or employee at a veterinary surgeon may dispense a prescription medicine for the treatment of an animal under the direction of the veterinary surgeon (Medicines Regulations (SR 1984/143) 7 August 2009; Regulation 42 (2)). This is in contrast to the tighter regulations in place in community pharmacy. Dispensary technicians, pharmacy graduates, pharmacy technicians and students can only dispense prescription medicines under the direct supervision of a pharmacist (Medicines Regulations (SR 1984/143) 7 August 2009; Regulation $42(1 \mathrm{~A})$ ) and all of their actions must be authorized as correct before the medication can leave the dispensary. Pharmacists also have ethical responsibilities to check that the doses are correct and the prescribed medicine is appropriate for the patient, before dispensing the medicine. If there is any uncertainty with these aspects or any component of the prescription, the pharmacist is obliged to contact the prescriber. In veterinary practice, as it is the veterinarian who prescribes the medicine and does the majority of the dispensing, they are responsible for ensuring that the dose and medicine is appropriate for their patients. Thus, for animals, the veterinarian carries out the roles of both doctor and pharmacist, which means there is no verification of dosage or suitability by an independent source. Pharmacists are in a suitable environment to provide medicines to the owners of animals, as they have both knowledge of medicines and access to supplies. Although we observed that it was not a common practice, there is definite potential for pharmacists to play a role in the veterinary field, as has been suggested by other authors. $^{4,7}$

In Dunedin, both veterinarian clinics and pharmacies source the majority of their medicines from the same supplier. This wholesaler does not restrict each professions to obtaining only medicines specific to their line of work, as both professions have access to both human and veterinary medicines. Where a product is not readily accessible, the veterinarians and pharmacists must either compound the product, order a suitable formulation or change the medication. The general consensus is that on-site compounding is being phased out in both professions in favor of manufactured formulations that can be ordered through compounding companies. In the case of veterinary medicine, compounding may be ordered specific to the patient through companies such as Optimus, a compounding pharmacy that specializes in extemporaneous compounding of both veterinary and human medicine. Pharmacists on the other hand are frequently required to compound these items for individual patients if the product is not readily available. 
The common classes of antimicrobials prescribed in a veterinary setting (Table 2) are also routinely prescribed for use in humans and are regularly dispensed in community pharmacies. This highlights the potential for pharmacist involvement in veterinary medicine with the provision of a more affordable and convenient service for animal owners. The emergence of antimicrobial resistance emphasizes the importance of ensuring the correct and appropriate use of antibiotics by veterinarians. ${ }^{8}$

With respect to labeling of medicines, the pharmacist must maintain a thorough, succinct process to produce a label adequate to the patient for both legal and ethical requirements. In veterinary practice, this process is less regulated with the labels being made either by the veterinarian at the time of dispensing or authorization or by verbal sanctioning to veterinary nurses. Labeling of veterinary medicine has additional requirements to prescribed medicines intended for human use. Table 3 shows the common requirements that are requisites for the labeling of medicines in both professions, and additional information that must be included in the veterinary setting. The difference evident with labeling of medicines intended for animals is the inclusion of the terms "For Animal Treatment Only" and "Keep Out of Reach of Children". This could be easily incorporated into the label when dispensed in the pharmacy setting, and is not a significant barrier that would prevent large quantities of animal medicines from being dispensed in a community pharmacy.

\section{CONCLUSIONS}

Veterinary and pharmacy professions carry out overlapping roles with respect to protocols and procedures regarding medication use in healthcare settings. Closer relationships between pharmacy and the veterinary profession been established in some countries, in particular the United Kingdom and the USA. For example, in the USA there are a number of compounding pharmacies for veterinary medicines and pharmacists on staff at veterinary hospitals across the country. Professional organizations, such as The Society of Veterinary
Hospital Pharmacists, the International College of Veterinary Pharmacy and Veterinary Pharmacists Group of the Royal Pharmaceutical Society of Great Britain have also been established to promote and enhance the continued development of veterinary pharmacy. In the United Kingdom, there are Prescribing Advisors who are pharmacists who work with general practitioners and primary care clinics to provide information on medicines management. The advice includes consistent best practice in prescribing and cost effective treatments that have the desired health outcomes for patients. We suggest that this is potentially a role that pharmacists could develop in association with veterinarians in New Zealand. The creation of such specialized positions would also require the education and training of pharmacists interested in the role of veterinary prescriber in subject areas including animal anatomy, physiology, pharmacokinetics, animal behavior ${ }^{3}$ and compounding for animal patients.

As pharmacists are the medication experts in the community, they are familiar with the different formulations and applications of medicines. In most instances, the medications used in the veterinary setting are the same as those used for the treatment of human conditions, and will not require an extensive expansion into different product lines as current human medications can be utilized within the different species. Integrating this knowledge with the veterinarians' extensive understanding of animals and animal conditions would create a vital link between the two professions, allowing pharmacists and veterinarians to work alongside each other to provide paramount health outcomes for their respective patients.

\section{ACKNOWLEDGEMENTS}

We are most grateful to the veterinary practices in Dunedin who enthusiastically participated in this study.

\section{CONFLICT OF INTEREST}

There were no conflicts of interest.

\section{References}

1. Prescott JF, Bailey J, Hagele WC, Leung D, Lofstedt J, Radostits OM, Sandals D. CVMA Task Force on "education, licensing, and the expanding scope of veterinary practice". Can Vet J. 2002;43:845-854.

2. Wick JY, Zanni GR. Patients large and small: role of the pharmacist in veterinary medicine. J Am Pharm Assoc. 2004;44:319-323.

3. Ceresia ML, Fasser CE, Rush JE, Scheife RT, Orcutt CJ, Michalski DI, Mazan MR, Dorsey MT, Bernardi SP. The role and education of the veterinary pharmacist. Am J Pharm Educ. 2009;73:1-10.

4. Karriker M, Wiebe V. Pharmacists in veterinary education: Bridging the gap. J Vet Med Educ. 2006;33:248-52.

5. Marsh J. Report of the independent review of dispensing by veterinary surgeons of prescription only medicines. In. London: MAFF Publications; 2001:65.

6. Arnold S, Gassner B, Giger T, Zwahlen R. Banning antimicrobial growth promoters in feedstuffs does not result in increased therapeutic use of antibiotics in medicated feed in pig farming. Pharmacoepidemiol Drug Saf. 2004;13:323331.

7. Caprile KA. Verterinary pharmacy. J Pharm Pract. 1989;2:83-91.

8. Maccabe AT, Matchett KF, Hueston WD. The need for public-health veterinarians as seen by future employers. J Vet Med Educ. 2008;35:269-274.

9. Kayne SB, Jepson MH. Veterinary Pharmacy. Chicago: Pharmaceutical Press; 2004. 